\title{
Hydrodynamic singularities and clustering in a freely cooling inelastic gas
}

\author{
Efi Efrati, Eli Livne and Baruch Meerson \\ Racah Institute of Physics, Hebrew University of Jerusalem, Jerusalem 91904, Israel
}

\begin{abstract}
We employ hydrodynamic equations to follow the clustering instability of a freely cooling dilute gas of inelastically colliding spheres into a well-developed nonlinear regime. We simplify the problem by dealing with a one-dimensional coarse-grained flow. We observe that at a late stage of the instability the shear stress becomes negligibly small, and the gas flows solely by inertia. As a result the flow formally develops a finite time singularity, as the velocity gradient and the gas density diverge at some location. We argue that flow by inertia represents a generic intermediate asymptotic of unstable free cooling of dilute inelastic gases.
\end{abstract}

PACS numbers: 45.70.Qj, 47.70.Nd

A gas of inelastically colliding macroscopic particles is a simple paradigm of granular matter [1, 2, 3, 4], and it appears in numerous applications, from astrophysics and geophysics to materials processing. One of the many fascinating phenomena in a freely cooling gas of inelastic particles is clustering instability 5, 6, 7, 8, 9, 10, 11, 12, 13]. This instability attracted much attention from physicists to rapid granular flow 3,14 . The clusters form an intricate cellular structure 6, 7, 8, 9, 11]. Though molecular dynamics (MD) simulations provide a valuable insight into the complicated dynamics of clustering, a better understanding requires a continuum theory. In this Letter we consider a freely cooling dilute gas of identical inelastic hard spheres. In this case a continuum theory is derivable systematically from a kinetic equation, leading to hydrodynamic equations with a heat loss term caused by the inelasticity of particle collisions 1, 15, 16, 17]. Hydrodynamics is expected to be an accurate leading order theory when the mean free path of the particles is much less than any length scale, and the mean time between two consecutive collisions is much less than any time scale, described hydrodynamically. Linearizing the hydrodynamic equations around a homogeneous cooling state (HCS), one finds two different linearly unstable modes: the shear mode and the thermal, or clustering, mode [6, 7, [8]. Growth of the shear mode corresponds to production of vorticity, while the clustering mode governs cluster formation. Nonlinear evolution of the clustering instability is a hard problem. Firstly, one has to deal here with nonlinear coupling of the shear and clustering modes. Secondly, as the local density grows, the hydrodynamic description becomes less accurate. It breaks down completely when the density approaches the point of the disorder-order transition in the gas of hard spheres.

In this Letter we follow the clustering instability into a well-developed nonlinear stage by circumventing these two difficulties. Firstly, we put the particles into a long two-dimensional (2D) box, $L_{x} \gg L_{y}$, so that the shear modes are strongly over-damped, and all coarse-grained quantities depend only on $x$. Secondly, we consider the limit of a very small area fraction of the particles. In this case, despite clustering, the gas density remains small, compared with the freezing density, for a very long time. Importantly, this limit does not preclude arbitrarily high density contrasts in the system. We solve the hydrodynamic equations numerically and observe that, at a late stage of the dynamics, the shear stress becomes negligibly small. As a result, the gas moves only by inertia, and the flow formally exhibits a finite time singularity. This singularity has a universal character if the initial mean velocity profile is smooth. We argue that flow by inertia is a generic intermediate asymptotic in more general multi-dimensional freely cooling granular flows, and that the finite time singularities form the skeleton of the later dynamics, when finite-density effects in the clusters come into play.

Let each of $N$ hard disks have a diameter $\sigma$ and mass $m=1$. Let the inelasticity of particle collisions be $q=(1-r) / 2>0$, where $r$ is the (constant) coefficient of normal restitution. Hydrodynamics deals with three coarse-grained fields: the number density $n(x, t)$, the mean velocity $v(x, t)$ and the granular temperature $T(x, t)$. We employ scaled variables $n \rightarrow n / n_{0}$, $T \rightarrow T / T_{0}, v \rightarrow v / T_{0}^{1 / 2}, x \rightarrow x / L_{x}$ and $t \rightarrow t T_{0}^{1 / 2} / L_{x}$, where $n_{0}=N /\left(L_{x} L_{y}\right)$ and $T_{0}$ are the average number density and the initial temperature of the gas, respectively. In the dilute limit, granular hydrodynamic equations 1, 15, 16, 17] read:

$$
\begin{aligned}
& \frac{d n}{d t}+n \frac{\partial v}{\partial x}=0, \quad \text { (a) } \quad n \frac{d v}{d t}-\frac{\partial P}{\partial x}=0, \quad \text { (b) } \\
& n \frac{d T}{d t}=P \frac{\partial v}{\partial x}+K \frac{\partial}{\partial x}\left(T^{1 / 2} \frac{\partial T}{\partial x}\right)-\frac{8 q}{K} n^{2} T^{3 / 2},
\end{aligned}
$$

where $d / d t=\partial / \partial t+v \partial / \partial x$ is the total time derivative, $K=(2 / \sqrt{\pi})\left(\sigma L_{x} n_{0}\right)^{-1}$ is the Knudsen number which, up to a constant factor of order unity, is the ratio of the mean free path of the particles to $L_{x}$, and $P=-n T+$ $(K / 4) T^{1 / 2}(\partial v / \partial x)$ is the stress field. The validity of Eqs. (11) and (2) requires $K \ll 1$ (scale separation), $n \sigma^{2} \ll 1$ (dilute limit), and $q \ll 1$ (nearly elastic collisions).

The HCS is described by Haff's cooling law $T(x, t)=$ $\left(1+t / t_{0}\right)^{-2}$, where $t_{0}=K / 4 q[1]$. A linear stability 
analysis of the HCS, analogous to that of Refs. [6, 7, 8], predicts clustering instability of the HCS if $K k_{x}<2 q^{1 / 2}$, where $k_{x}$ is the (scaled) wave number of a small sinusoidal perturbation around the HCS. The wave number is quantized by the periodic boundary conditions: $k_{x}=2 \pi k$, where $k=1,2, \ldots$ is the mode number. Therefore, the $k$ th mode is linearly unstable if $\pi k K<q^{1 / 2}$ [18]. The rest of the parameters fixed, the instability occurs when $L_{x}$ is sufficiently large. The number of the unstable modes in the system $k_{\max }$ can serve as a measure of the instability magnitude. The growth/decay of small perturbations is algebraic. The density perturbations grow. The temperature and velocity perturbations decay, but the decays are slow compared to Haff's cooling law. As a result, the flow tends to become supersonic [8].

We followed the clustering instability with $k_{\max } \gg 1$ into a strongly nonlinear regime by solving Eqs. (11) and (2) numerically. We used a Lagrangian scheme 19] with periodic boundary conditions. The Lagrangian description allowed us to resolve steep velocity gradients and high density peaks with good accuracy until close to singularities, see below. The first series of hydrodynamic simulations dealt with generic initial conditions of the form $n(x, t=0)=1+\delta n(x), T(x, t=0)=1+\delta T(x)$ and $v(x, t=0)=\delta v(x)$, where each of the small terms $\delta n(x), \delta T(x)$ and $\delta v(x)$ is a sum of a few hundred Fourier modes with random small amplitudes, of which a few dozen modes are linearly unstable. In all these simulations we observed strong clustering: development of multiple high and narrow density peaks, accompanied by steepening velocity gradients, as the gas temperature continues to decay. The gas density in the peaks grows without limit, until the time when our finite-difference scheme is unable to accurately follow the density growth in the highest density peak. The temporal growth of the density peaks, and of the velocity gradients, accelerates rapidly, implying a finite-time singularity. Figure 1 shows a typical snapshot of the system close to the time of singularity.

A convenient integral measure of the unstable cooling dynamics is the total energy of the system:

$$
E(t)=\int_{-1 / 2}^{1 / 2}\left(n T+\frac{1}{2} n v^{2}\right) d x
$$

where $n T$ is the thermal energy density, and $n v^{2} / 2$ is the macroscopic kinetic energy density. A plot of $E(t)$ is shown in Fig. 2. As expected, $E(t)$ follows Haff's law at early times, but deviates from it at later times. Figure 2 elucidates the role of each of the two terms in Eq. (3). Both the thermal energy, and the macroscopic kinetic energy initially decay with time; the thermal energy decays faster. At later times the kinetic energy approaches a constant. As a result, $E(t)$ is dominated by the thermal energy at early times and by the kinetic energy at later times. Remarkably, the thermal energy continues

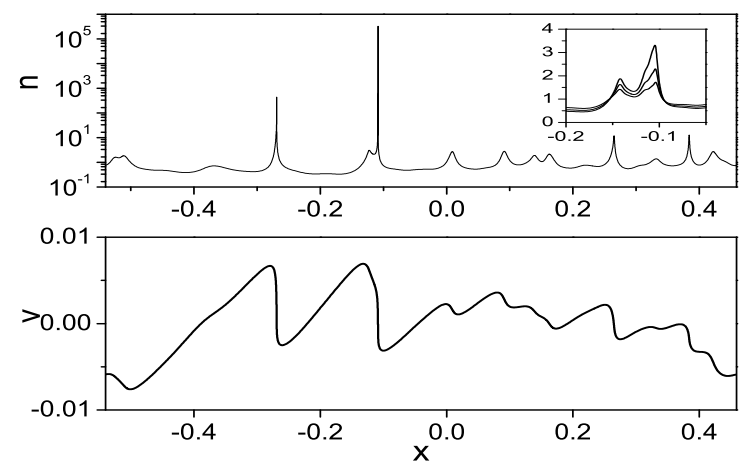

FIG. 1: The density and velocity profiles at scaled time $t=7.043$, shortly before the major density peak develops singularity. The parameters $K=4 \cdot 10^{-4}$ and $q=10^{-2}$ correspond to 79 linearly unstable Fourier modes. 2000 Lagrangian mesh points are used, so the major density peak includes more than 50 mesh points above the density value of $n=10^{2}$. The inset shows an earlier density history (at $t=2,3$ and 4 ) of a region around the major density peak.

to follow Haff's law until the time of singularity.

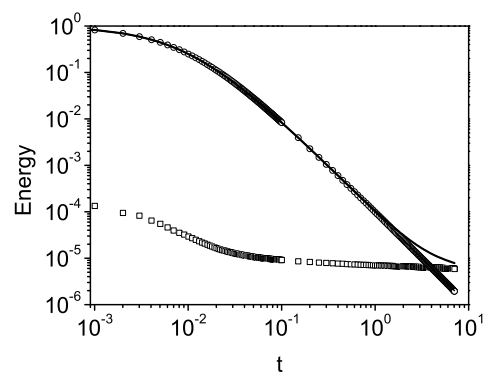

FIG. 2: The total energy of the system $E$ (thick solid line), the thermal energy (circles), the macroscopic kinetic energy (squares) and Haff's cooling law (thin solid line) versus time for the simulation shown in Fig. 1.

The finite-time singularities of the velocity gradient and the density strongly indicate that, at late times, the stress tensor $P$ becomes negligibly small, and the gas flows by inertia only. An additional evidence for the flow by inertia is the constancy of the macroscopic kinetic energy at late times. The flow by inertia is described by the equation

$$
\frac{\partial v}{\partial t}+v \frac{\partial v}{\partial x}=0
$$

and Eq. (1)a. This problem is soluble analytically [20]:

$$
v(x, t)=v_{0}(\xi), \quad(a) \quad n(x, t)=\frac{n_{0}(\xi)}{1+t v_{0}^{\prime}(\xi)} .
$$

where $v_{0}^{\prime}(\xi)=d v_{0}(\xi) / d \xi$, while $v_{0}(\xi)$ and $n_{0}(\xi)$ are the velocity and density of the gas, respectively, at some "initial" moment of time (which should be late enough so 
that the flow by inertia has already set in). The relation between Eulerian coordinate $x$ and Lagrangian coordinate $\xi$ is the following: $x=\xi+v_{0}(\xi) t$. The finite-time singularities of both the velocity gradient

$$
\frac{\partial v(x, t)}{\partial x}=\frac{v_{0}^{\prime}(\xi)}{1+t v_{0}^{\prime}(\xi)},
$$

and the density, Eq. (5)b, occur when the denominator in Eq. (6) becomes zero for the first time. We compared these predictions with a numerical solution of the full hydrodynamic equations (11) and (2), for the same parameters $K=4 \cdot 10^{-4}$ and $q=10^{-2}$, but with simpler initial conditions: $n(x, t=0)=T(x, t=0)=1$, and a single Fourier mode for the velocity:

$$
v(x, t=0)=a \sin (2 \pi x), \quad a=-0.05 .
$$

In this case only one singularity develops (at $x=0$ ). Figure 3 shows the gas velocity $v$ versus $\xi=x-t v(x, t)$ at different times. The different curves collapse into a single curve with an accuracy better than $1.5 \%$. Additional tests deal with the behavior of the flow in the close vicinity of $x=0$, as the singularity time is approached. For this smooth symmetric flow we can write $v_{0}(\xi)=-\xi / \tau+C \xi^{3}+\mathcal{O}\left(\xi^{5}\right)$, where $t=\tau$ is the time of singularity in the flow-by-inertia model, and $C>0$ is a constant. In the Eulerian coordinates this yields a solution in an implicit form. In the leading order

$$
x=-t^{\prime} v\left(x, t^{\prime}\right)-C \tau^{4} v^{3}\left(x, t^{\prime}\right),
$$

where $t^{\prime}=\tau-t$ is the time to the singularity. Not too close to the singularity point $x=0$ one obtains $v \sim(-x)^{1 / 3}$. As the velocity profile (8) is self-similar: $v\left(x, t^{\prime}\right)=\left(t^{\prime}\right)^{1 / 2} V\left[x /\left(t^{\prime}\right)^{3 / 2}\right]$, the velocity gradient is $\partial v / \partial x=\left(t^{\prime}\right)^{-1} d V / d w$, where $w=x /\left(t^{\prime}\right)^{3 / 2}$. The shape function $V(w)$ is determined by the equation $C \tau^{4} V^{3}+$ $V+w=0$. What is the density behavior close to the singularity? Very close to $x=0$ the density grows indefinitely: $n_{0}(0)(1-t / \tau)^{-1}$; outside of that region (but still close enough to $x=0) n(x, t)$ approaches a universal profile $n \sim|x|^{-2 / 3}[21]$. We verified these properties numerically, see examples in Fig. 4. Importantly, for a strong instability, $k_{\max } \gg 1$, the system "freezes up", and the motion by inertia sets in very rapidly. Indeed, the scaled velocity profile in Fig. 3 is very close to the initial profile (7). Building on this simplification, we can expand Eq. (7) in the vicinity of $x=0$ and predict the time of singularity: $\tau=(2 \pi|a|)^{-1} \simeq 3.18$ which agrees within $2 \%$ with the simulation result, see Fig. 4a. In addition, the linear time dependence of the quantities, shown in the inset of Fig. 4a, sets in already at early times.

Therefore, a strongly nonlinear regime of the quasione-dimensional clustering instability in a dilute granular flow is describable by a simple flow by inertia, until the

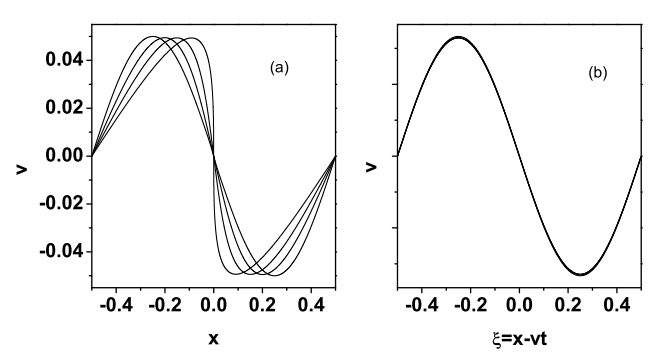

FIG. 3: The numerically computed velocity is shown versus $x$ (a) and versus $\xi=x-v t$ (b) at times 1,2 and 3.225 (the profiles in figure a steepen as the time progresses). Also shown in figure b is the initial profile (7). All the curves in figure b coincide within $1.5 \%$. The simulation parameters are $K=4 \cdot 10^{-4}$ and $q=10^{-2}$.
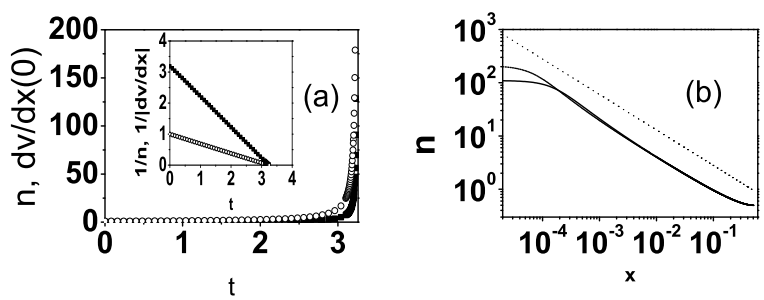

FIG. 4: Numerically computed values of $|\partial v / \partial x|$ (filled squares) and $n$ (empty circles) at $\mathrm{x}=0$ versus time (a). The inset shows the respective inverse values. Figure b depicts the spatial profiles of $n$ at time moments 3.209 and 3.225. The straight line is a $x^{-2 / 3}$ dependence; it is given for reference. The parameters are the same as in Fig. 3.

moment of singularity [22]. In a related work Ben-Naim et al. 23] investigated the dynamics of point-like particles, inelastically colliding on a line. The strictly onedimensional setting of Ref. 23] makes a hydrodynamic description problematic. Still, Ben-Naim et al. observed that the Burgers equation with vanishing viscosity is a proper continuum model for their system. It remains to be seen whether the Burgers equation or some other saturation mechanism applies to our quasi-one-dimensional model at a later stage of the dynamics, when finitedensity effects come into play. We stress that the (hydrodynamic) density singularities are entirely different from inelastic collapse 24] (divergence of the particle collision rate at some locations) which is a discrete-particle effect. We also note in passing that statistical properties of the flow by inertia (for example, the dynamics of the structure function) are well understood 25].

What can be said about a fully multi-dimensional strongly unstable cooling flow, when unstable shear and clustering modes are coupled? A natural assumption, motivated already by the linear theory of the clustering/shearing instabilities of the HCS [6, 7, 8], is that the stress tensor "freezes up", and flow by inertia sets in 
here as well. A possible counter-argument involves viscous heating of the system by the unstable shear modes. The heating effect is absent in the linear regime of the instability (as the viscous heating is of the second order with respect to the perturbation amplitude), but it comes into play in the nonlinear regime. The present state of theory makes it difficult to prove that the viscous heating cannot arrest, in some locations, the freezing of the stress tensor. However, MD simulations in 2D strongly indicate that the freezing continues unarrested. For example, Nie et al. [12] observe that, at late times, "the thermal energy becomes much smaller than the (macroscopic) kinetic energy". Based on this evidence we argue that, in the dilute regime, this strongly supersonic flow should be describable by multi-dimensional flow-by-inertia equations

$$
\partial \mathbf{v} / \partial t+(\mathbf{v} \cdot \nabla) \mathbf{v}=\mathbf{0}, \quad \partial n / \partial t+\nabla \cdot(n \mathbf{v})=0
$$

This flow also exhibits finite-time singularities [21, 26], and the singularities form cellular structures, most of the material being concentrated along the cell boundaries 21]. This picture resembles the density distribution of granular clusters observed in MD simulations of freely cooling gases of inelastic hard spheres in $2 \mathrm{D}$ 6, 7, 8, 9, 11]. Interestingly, the multi-dimensional singularities of Eqs. (9) were studied previously in an entirely different context: in the so called Zeldovich approximation of theory of formation of structure in an expanding universe [21].

We stress that there are important differences between the multi-dimensional clustering instability and the Zeldovich model. In the process of clustering instability of inelastic gases a considerable vorticity is generated, while in Zeldovich model the flow is assumed to be potential 21]. Still, it was found, in a rare treatment of a more general (non-potential) velocity field, that "high-density regions should be high-vorticity regions" [27]. This finding appears to agree with MD simulations of freely cooling granular gases in 2D 7].

In summary, by following the unstable cooling dynamics of a dilute inelastic gas we identified an important new intermediate asymptotic regime: a nonlinear flow by inertia. We argue that high-density regions in the gas, which are precursors of densely packed granular clusters, are caused by the flow by inertia, rather than directly by the pressure gradient. Our results indicate that the role of the clustering and shearing instabilities of the free cooling is "merely" to produce a long-lived spatially non-uniform supersonic velocity field needed for the development of the high-density regions by the flow by inertia. Therefore, a due account of the flow-by-inertia regime will be important in the future theory of "life after singularity", where the singularities are smoothed by finite-density effects in the clusters, and a coarsening process develops 11. No first-principles coarse-grained description of that final stage is yet available.
We thank A. Puglisi, P. V. Sasorov and S. F. Shandarin for useful discussions. This research was supported by the Israel Science Foundation.

[1] P.K. Haff, J. Fluid Mech. 134, 401 (1983).

[2] C.S. Campbell, Annu. Rev. Fluid Mech. 22, 57 (1990).

[3] L.P. Kadanoff, Rev. Mod. Phys. 71, 435 (1999).

[4] I. Goldhirsch, Annu. Rev. Fluid Mech. 35, 267 (2003).

[5] M.A. Hopkins and M.Y. Louge, Phys. Fluids A 3, 47 (1991).

[6] I. Goldhirsch and G. Zanetti, Phys. Rev. Lett. 70, 1619 (1993); I. Goldhirsch, M.-L. Tan, and G. Zanetti, J. Sci. Computing 8, 1 (1993); N. Sela, I. Goldhirsch, and S.H. Noskovicz, Phys. Fluids 8, 2337 (1996).

[7] S. McNamara, Phys. Fluids A 5, 3056 (1993); S. McNamara and W.R. Young, Phys. Rev. E 53, 5089 (1996).

[8] P. Deltour and J.-L. Barrat, J. Phys. I. France 7, 137 (1997).

[9] R. Brito and M. H. Ernst, Europhys. Lett. 43, 497 (1998).

[10] J.J. Brey, M.J. Ruiz-Montero, and D. Cubero, Phys. Rev. E 60, 3150 (1999).

[11] S. Luding and H. J. Herrmann, Chaos 9, 673 (1999).

[12] X.B. Nie, E. Ben-Naim and S.Y. Chen, Phys. Rev. Lett. 89, 204301 (2002).

[13] Similar condensation instabilities in optically thin plasmas are driven by radiative cooling. For a review see B. Meerson, Rev. Mod. Phys. 68, 215 (1996).

[14] H.M. Jaeger, S.R. Nagel, and R.P. Behringer, Rev. Mod. Phys. 68, 1259 (1996); Physics Today 49 (4), 32 (1996).

[15] J.T. Jenkins and M.W. Richman, Phys. Fluids 28, 3485 (1985).

[16] N. Sela and I. Goldhirsch, J. Fluid Mech. 361, 41 (1998).

[17] J.J. Brey, J.W. Dufty, C.S. Kim, and A. Santos, Phys. Rev. E 58, 4638 (1998).

[18] To suppress shear modes and clustering modes in the $y$ direction, we impose reflecting boundary conditions at $y=0$ and $y=L_{y}$ and demand $q^{1 / 2} \ll(\pi / 4) K\left(L_{x} / L_{y}\right)$.

[19] R.D. Richtmyer and K.W. Morton, Difference Methods for Initial Value Problems (Interscience, New York, 1967).

[20] G.B. Whitham, Linear and Nonlinear Waves (Wiley, New York, 1974), Chapter 2.

[21] S.F. Shandarin and Ya. B. Zeldovich, Rev. Mod. Phys. 61, 185 (1989).

[22] The quasi-one-dimensional regime can be probed in MD simulations in $2 \mathrm{D}$. The parameters must be chosen so that the gas is dilute, the clustering instability in the $x$ direction is strong, and the shear/clustering instabilities in the $y$-direction are strongly suppressed [18].

[23] E. Ben-Naim, S.Y. Chen, G.D. Doolen, and S. Redner, Phys. Rev. Lett. 83, 4069 (1999).

[24] S. McNamara and W.R. Young, Phys. Rev. E 50, 28R (1994).

[25] S.N. Gurbatov, A.N. Malakhov, and A.I. Saichev, Nonlinear Random Waves and Turbulence in Nondispersive Media: Waves, Rays, Particles (Manchester University Press, Manchester, 1991), Chapter 4.

[26] V.I. Arnold, Catastrophe Theory (Springer, Berlin, 1992); J.W. Bruce, J. London Math. Soc. 33 (2), 375 (1986). 
[27] J.D. Barrow and P. Saich, Class. Quantum Grav. 10, 79 (1993). 